\title{
Prognostic and predictive value of immunological parameters for chemoradioimmunotherapy in patients with pancreatic adenocarcinoma
}

\author{
S Karakhanova ${ }^{1}$, E Ryschich ${ }^{1}$, B Mosl ${ }^{1}$, S Harig ${ }^{1,2}$, D Jäger ${ }^{2}$, J Schmidt ${ }^{1,3}$, W Hartwig ${ }^{4}$, J Werner ${ }^{4}$ and A V Bazhin ${ }^{* 1,4}$ \\ ${ }^{1}$ Department of General Surgery, University Hospital Heidelberg, 69120 Heidelberg, Germany; ${ }^{2}$ National Centre for Tumor \\ Diseases, University Hospital Heidelberg, 69120 Heidelberg, Germany; ${ }^{3}$ General and Visceral Surgery Center, 8002 Zurich, \\ Switzerland and ${ }^{4}$ Department of General, Visceral, Transplantation, Vascular and Thoracic Surgery, Hospital of the University \\ of Munich, 81377 Munich, Germany
}

Background: Chemoradioimmunotherapy of patients with pancreatic adenocarcinoma from the CapRI trial did not show any benefit of interferon- $\alpha$ in addition to a 5-fluorouracil (5FU)-based treatment. The aim of this study was to identify immunological parameters in patients from this trial to be used for predictive and/or prognostic purposes.

Methods: The following methods were used: tumour immunohistology, FACS analyses, cytokine measurement, as well as cytotoxicity and ELIspot. Immunological parameters were correlated with patients' survival using the Kaplan-Meier method.

Results: Irrespective of therapy type, high lymphocyte accumulation in tumours and frequencies of NK cells and effector (eff) $\mathrm{CD}^{+}{ }^{+} \mathrm{T}$ cells in peripheral blood of the patients were associated with patients' survival. Amount of CD3 ${ }^{+}$and effector-memory $\mathrm{CD}^{+}$blood lymphocytes, expression of CD152 and interleukin (IL)-2 serum level showed a predictive value for chemoradioimmunotherapy. Tumoural accumulation of $\mathrm{CD}^{+}$and $\mathrm{CD}^{+}$cells was predictive for outcome of chemotherapy alone. Besides, we identified the frequencies of $\mathrm{CD}^{+}$lymphocytes, effCD8 ${ }^{+} \mathrm{T}$ cells and NK cells in the peripheral blood of the patients, and IL-10 amount in serum, to be predictive values for 5FU-based chemotherapy.

Conclusions: Immunological parameters, identified in this trial as possible markers, may be of interest in personalized medicine towards the improvement of the treatment and prognosis of pancreatic carcinoma patients.

The prognosis of patients with carcinoma of the exocrine pancreas is comparatively poor. Even in the case of resectable disease, the 5-year survival rate is $<5 \%$ with a median survival of 6 months without an adjuvant treatment (Cress et al, 2006). Adjuvant chemotherapy based on gemcitabine or 5-fluorouracil (5FU) demonstrated a significant increase in overall survival (OS) and disease-free survival (DFS; Oettle et al, 2007; Neoptolemos et al, 2010). Presently, a combination of different chemotherapeutics with the goal of improving the efficacy of chemotherapy is under clinical investigation. Recently published data from the FOLFIRINOX chemotherapeutic trial revealed a significant survival advantage in the palliative setting, but this was associated with an increased toxicity (Conroy et al, 2011). Likewise, a newly conducted phase III clinical study in patients with metastatic disease treated with nabpaclitaxel combined with gemcitabine showed a significantly improved OS rate compared with patients treated with gemcitabine alone; however, increased rates of myelosuppression and peripheral neuropathy were documented (Von Hoff et al, 2013).

There is growing evidence, particularly from preclinical studies, that chemotherapy combined with immunotherapy may improve 
drug efficiency against pancreatic cancer and other types of cancers (Lake and Robinson, 2005). This fact prompted us to validate the clinical impact of this regimen through a randomized phase III study (the CapRI trial) in which the combined treatment interferon- $\alpha$ (IFN), $5 \mathrm{FU}$ and beam radiation - was compared with 5 FU-based chemotherapy alone in the adjuvant setting (Knaebel et al, 2005). The clinical data from the CapRI trial did not show any significant improvement in patients' survival compared with chemotherapy alone (Schmidt et al, 2012). However, the clinical outcome in both groups represented the best-ever reported survival for patients with resected pancreatic cancer in a phase III controlled randomized trial (26.5 months for chemoradioimmunotherapy vs 28.5 months for chemotherapy alone).

Most cancer therapeutics have limited benefit for the whole patient population; hence, personalized medicine using biomarkerguided pre-selection of patients may be promising for the improvement of cancer therapy (Simon, 2008). A cancer biomarker can be defined as a biological feature that provides diagnostic, prognostic, predictive, or therapeutic information about a particular disease or subject (Jimeno and Hidalgo, 2006). A predictive biomarker should indicate the differential efficacy (benefit) of a particular therapy based on marker status, whereas a prognostic marker identifies patients with differing risks of a specific outcome, such as progression or death (Simon and Altman, 1994). A prognostic marker can stratify a population of patients into groups in whom different treatment options are appropriate, but it cannot guide the choice of a particular therapy. Immunological parameters such as the number of immune cells, their phenotype and activation state, serum cytokine concentration and others could serve as prognostic and/or predictive biomarkers, especially in the context of cancer immunotherapy (Copier et al, 2006; Bellati et al, 2009; Gogas and Kirkwood, 2009).

In this work we retrospectively evaluated immunological parameters and their association with DFS and OS for chemoradioimmunotherapy, with IFN- and 5FU-based chemotherapy alone, in pancreatic carcinoma patients enroled in the multicentre phase III CapRI study.

\section{MATERIALS AND METHODS}

Patients' characteristics, study set-up and sample preparation. The final protocol was approved by the ethics committee of the University of Heidelberg, Medical School, Heidelberg, Germany (L-042/2003). Informed consent was obtained from all patients included in the study. Trial Registration: Current Controlled Trials ISRCTN62866759. The trial set-ups were recently published (Schmidt et al, 2012). Demographics and clinical characteristics of CapRI patients involved in this immunological study are summarized in the Table 1. Peripheral blood mononuclear cells (PBMCs) were isolated from heparinized blood samples by Ficoll density gradient centrifugation. The cells were frozen in 10\% DMSO (Sigma, Munich, Germany) and 20\% FCS (PAA, Cölbe, Germany), and the serum samples were stored at $-80^{\circ} \mathrm{C}$. EDTA blood was used immediately for flow cytometric analysis. For cytotoxicity assays, the monocytes were removed after thawing, by adherence to plastic.

Immunohistology. For immunohistological analysis of tumoural $\mathrm{T}$-cell accumulation, the frozen preps of resected pancreatic tissues were used. Sections of $5 \mu \mathrm{m}$ were cut, air-dried and fixed in acetone. The slides were stored at $-20^{\circ}$ until further use. The sections were stained by indirect three-step immunohistochemistry using the LSAB-kit (Dako, Hamburg, Germany) and counterstained using Mayer's acid hemalum (Fluka, Steinheim, Germany). The following clones of purified monoclonal antibodies were used: F7.2.38 (anti-CD3 and T cells), MT310 (anti-CD4) and DK25
Table 1. Demographics and clinical characteristics of patients from the study

\begin{tabular}{|c|c|c|c|c|c|c|}
\hline \multirow{3}{*}{ Characteristics } & \multicolumn{2}{|c|}{ Arm A } & \multicolumn{2}{|c|}{ Arm B } & \multicolumn{2}{|c|}{ Both Arms } \\
\hline & $\begin{array}{c}\text { No. of } \\
\text { patients }\end{array}$ & $\%$ & $\begin{array}{c}\text { No. of } \\
\text { patients }\end{array}$ & $\%$ & $\begin{array}{c}\text { No. of } \\
\text { patients }\end{array}$ & $\%$ \\
\hline & 48 & 52 & 44 & 48 & 92 & 100 \\
\hline \multicolumn{7}{|l|}{ Age, years } \\
\hline $\begin{array}{l}\text { Mean } \\
\text { Range }\end{array}$ & $\begin{array}{c}60.7 \\
37.0-74.0\end{array}$ & & $\begin{array}{c}62.6 \\
33.0-77.0\end{array}$ & & $\begin{array}{c}61.6 \\
33.0-77.0\end{array}$ & \\
\hline \multicolumn{7}{|l|}{ Sex } \\
\hline $\begin{array}{l}\text { Female } \\
\text { Male }\end{array}$ & $\begin{array}{l}17 \\
31\end{array}$ & $\begin{array}{l}35 \\
65\end{array}$ & $\begin{array}{l}23 \\
21\end{array}$ & $\begin{array}{l}52 \\
48\end{array}$ & $\begin{array}{l}40 \\
52\end{array}$ & $\begin{array}{l}44 \\
56\end{array}$ \\
\hline \multicolumn{7}{|l|}{ Resection } \\
\hline $\begin{array}{l}\text { R0 } \\
\text { R1 }\end{array}$ & $\begin{array}{l}27 \\
21\end{array}$ & $\begin{array}{l}56 \\
44\end{array}$ & $\begin{array}{l}25 \\
19\end{array}$ & $\begin{array}{l}57 \\
43\end{array}$ & $\begin{array}{l}52 \\
40\end{array}$ & $\begin{array}{l}57 \\
43\end{array}$ \\
\hline
\end{tabular}

\begin{tabular}{|l|r|r|r|r|r|r|}
\hline \multicolumn{7}{|l|}{ Tumour status } \\
\hline T1 & 1 & 2 & 0 & 0 & 1 & 1 \\
T2 & 2 & 4 & 0 & 0 & 2 & 2 \\
T3 & 45 & 94 & 43 & 98 & 88 & 96 \\
T4 & 0 & 0 & 1 & 2 & 1 & 1 \\
\hline Node status & 10 & 21 & 8 & 18 & 18 & 20 \\
N0 & 38 & 79 & 36 & 82 & 74 & 80 \\
N1 & 2 & 4 & 1 & 2 & 3 & 3 \\
\hline Grade & 31 & 65 & 32 & 73 & 63 & 69 \\
\hline 1 & 15 & 31 & 11 & 25 & 26 & 28 \\
2 &
\end{tabular}

(anti-CD8). All antibodies were purchased from Dako GmbH (Hamburg, Germany). Quantitative analysis of immunohistochemical staining was performed by computer-assisted image analysis. For this aim, the microscopic fields were randomly chosen by a light microscope, digitized by video camera and saved on a computer. The number of positively stained lymphocytes in one random microscopic field (surface $0.105 \mathrm{~mm}^{2}$ ) was counted using Histo software (Dept. Exp. Surgery, Univ. Heidelberg, Germany).

Flow cytometric analysis. Flow cytometric analysis was performed as described elsewhere (Karakhanova et al, 2014). Leukocytes were characterized using the following fluorescence labelled anti-human antibodies: CD45 (for all leukocytes), CD3, CD152 (CTLA4), CD56, NKG2D, CD69, CD8, CD45RA, CCR7, CD27, CD33, CD14, CD80, HLA-DR and CD21 (all from BD Biosciences, Heidelberg, Germany; Schmidt et al, 2007). For FACS analyses, antibodies were placed into a $5-\mathrm{ml}$ polystyrene roundbottom tube and $100 \mu$ l blood was added, briefly shaken and incubated for $15 \mathrm{~min}$ at room temperature. Afterwards, $2 \mathrm{ml} 1 \times$ BD FACS Lysing Solution (BD Biosciences) was added and further incubated for another $10 \mathrm{~min}$ at room temperature, followed by centrifugation at $200 \mathrm{~g}$ for $5 \mathrm{~min}$. The supernatant was removed, the pellet re-suspended in $2 \mathrm{ml}$ Cellwash solution (BD Biosciences) and centrifuged again. Cells were re-suspended in $250 \mu \mathrm{l}$ Cellwash and measured on Epics XL-MCL (Beckman Coulter, Munich, Germany).

Analysis of cytokines with FlowCytomix and cytotoxicity assay. Serum cytokines were determined using the FlowCytomix technology (Bender Med System, Vienna, Austria) and a standard chromium release assay was used to determine cytotoxic activity. Both methods have been described previously in Schmidt et al (2007).

ELIspot. For analysis of granzyme $\mathrm{B}$ release the BD ELISPOT Human Granzyme B ELISPOT Set (BD Bioscience, San Jose, CA, USA) was used according to the manufacturer's instructions. Patients' PBMCs were stimulated with $100 \mu \mathrm{g} \mathrm{ml}^{-1} \mathrm{MUC1}-$ made 
as described in Schmidt et al (2007) - or $100 \mathrm{Uml}^{-1}$ CA19.9 (Merck, Schwalbach, Germany), or with medium as a control. Spots were counted after $24 \mathrm{~h}$ of incubation using the KS ELISPOT System, release 4.1 (Carl Zeiss Light Microscopy, Jena, Germany). Cells stimulated with medium without the peptides served as controls.

Statistical analysis. GraphPad Prism Version 5.01 software (GraphPad Software, La Jolla, CA, USA) was used for all statistical analyses. Distributions of continuous variables were presented as symbol and line plots. Missing data were not replaced or imputed. D'Agostino and Pearson omnibus normality test were conducted to estimate the distribution of data. The null hypothesis (mean values were equal) $v s$ the alternative hypothesis (mean values were not equal) was tested by Wilcoxon matched pairs test for the data that did not pass the normality test. The median of the immunological parameters was used as a cut-off to discriminate between the high parameter level (value as the cut-off) from the low level (value $<$ as the cut-off). For some parameters, as indicated in the figures and text, cut-off was determined as present or absent in the parameter. Overall survival was calculated from the date of surgery until the date of death. Disease-free survival was calculated from the date of surgery until the date of relapse (local or metastatic). Survival probabilities were estimated using the Kaplan-Meier method. The null hypothesis (survival curves equal) vs the alternative hypothesis (survival curves not equal) was tested by the log-rank (MantelCox) and the Wilcoxon-Gehan (Gehan-Breslow-Wilcoxon) tests. Hazard ratios with 95\% CIs were determined using the MantelHaenszel method. The significance level was $\alpha=5 \%$.

\section{RESULTS}

Possible prognostic immunological markers for patients with pancreatic cancer. To investigate a possible prognostic value of immunological parameters for pancreatic cancer independent of therapy carried out, we performed a survival analysis (DFS and OS) depending on the immune state of each patient before treatment.

Immunohistochemical analysis of $\mathrm{T}$ cells in tumours revealed accumulation of $\mathrm{T}$ lymphocytes to be positively correlated with better OS and DFS of patients (cut-off $267 \mathrm{CD}^{+} \mathrm{T}$ cells, 229 $\mathrm{CD}^{+}$cells and $95 \mathrm{CD}^{+}$cells per total area of $1 \mathrm{~mm}^{2}$; Figure 1 and Supplementary Table S1). FACS analysis of the patients' peripheral blood showed neutrophil quantity to have a tendency to correlate negatively with patients' DFS (cut-off 50.9\% of PBMC; Supplementary Table S1). Surprisingly, a negative correlation was detected between frequency of NK cells and OS (cut-off $20.85 \%$ of leukocytes; Figure 2 and Supplementary Table S1). The amount of

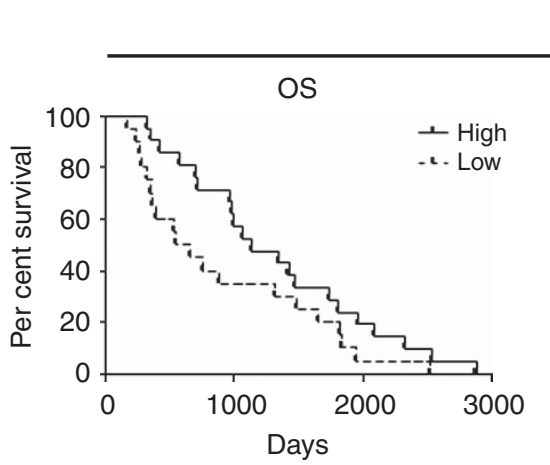

CD3
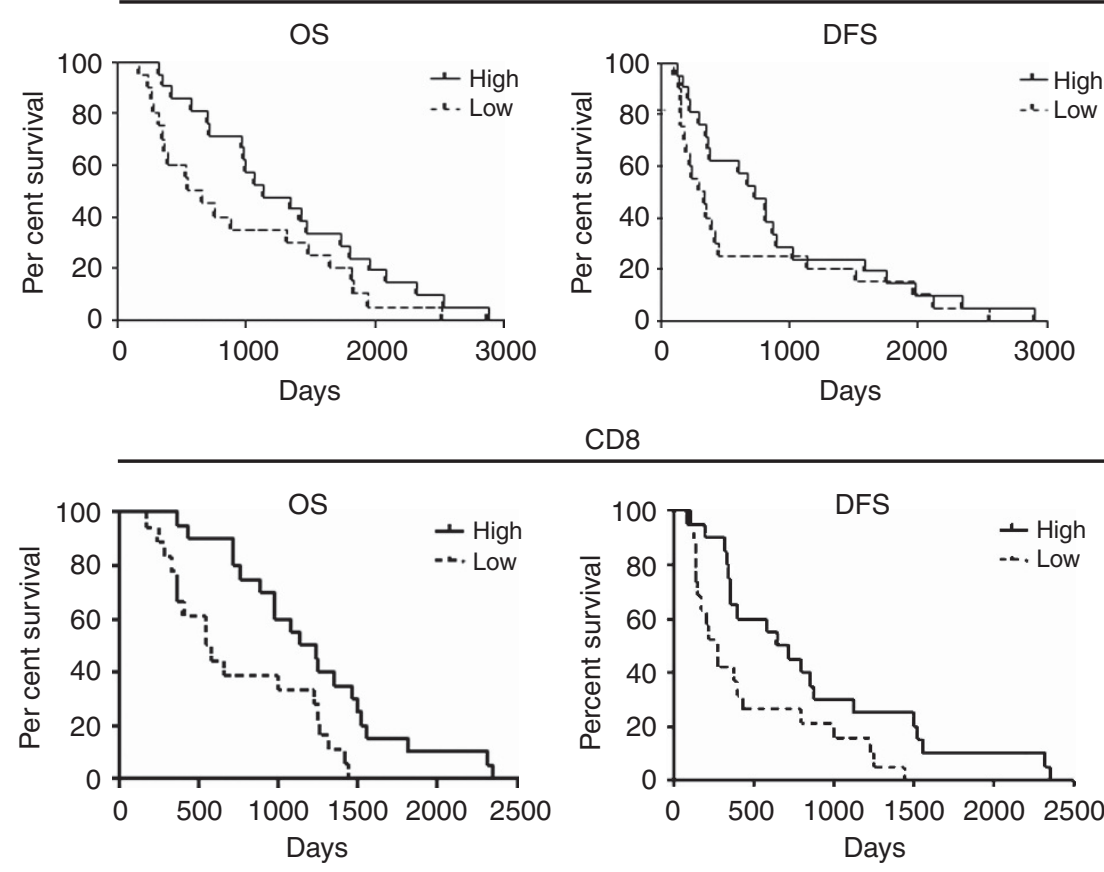

CD8
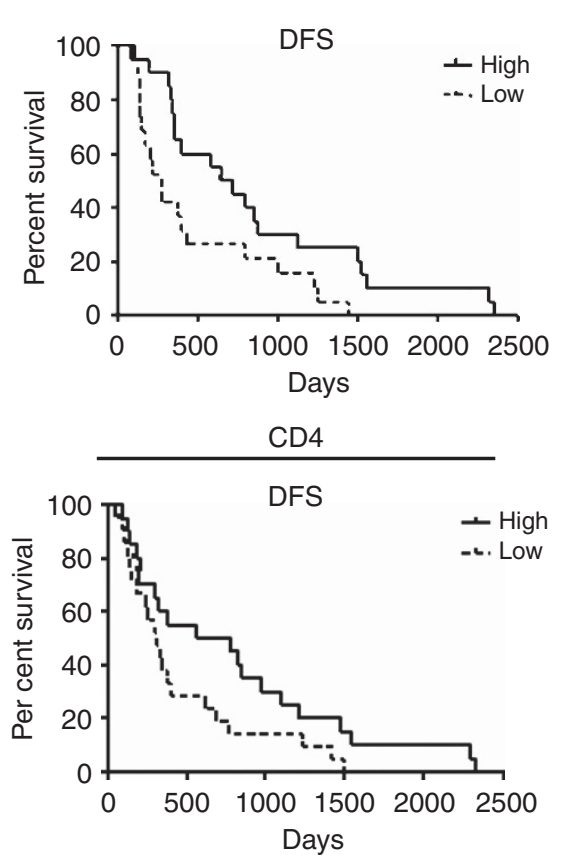

Figure 1. Accumulation of lymphocytes as a prognostic parameter for patients with pancreatic cancer. Tumour tissues of patients from both study arms were immunohistologically investigated, as mentioned in the Materials and Methods section, and data were analysed with Kaplan-Meier curves. For statistical data, see Supplementary Table S1. 


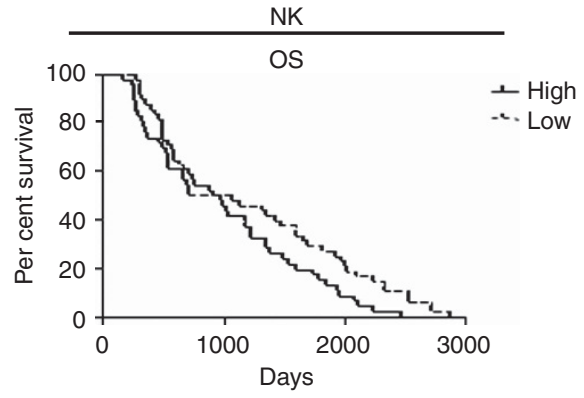

Effector CD8

OS

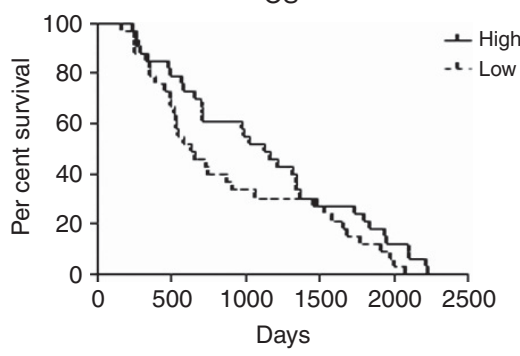

Granzyme B release (CA19)

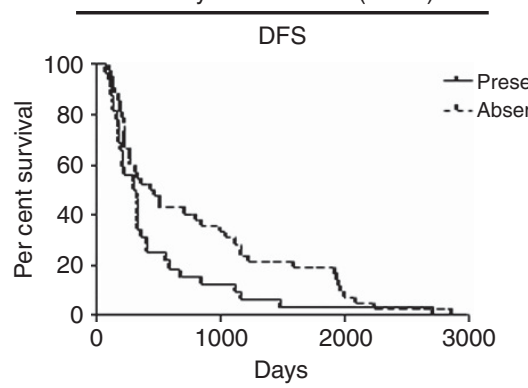

DFS

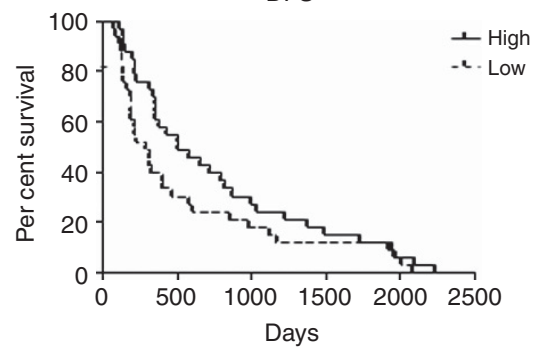

Granzyme B release (MUC)

DFS

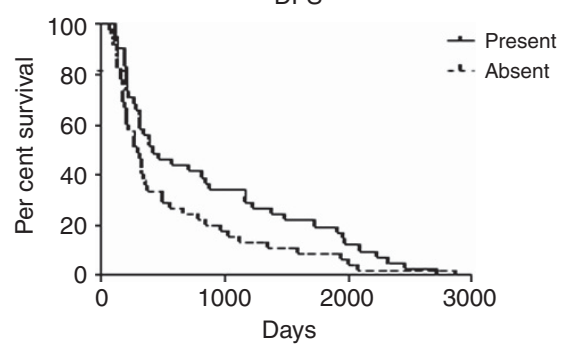

Figure 2. Immunological parameters measured in the peripheral blood of patients with pancreatic cancer in the prognostic setting. FACS analysis of immune cells and granzyme B release were assessed as described in the Materials and Methods section and the data were correlated with patients' OS and/or DFS with Kaplan-Meier methods. For statistical data, see Supplementary Table S1.

effector (eff) $\mathrm{CD}^{+} \mathrm{T}$ cells showed a positive correlation with patients' survival (cut-off $14.8 \%$ of $\mathrm{CD}^{+}$T cells; Figure 2 and Supplementary Table S1). At the same time, the cytotoxic activity of $\mathrm{CD}^{+} \mathrm{T}$ cells, measured by granzyme B release, demonstrated different directions of correlation depending on the antigen used: patients with detectable granzyme B release after stimulation with MUC1 antigen had a better DFS compared with patients without the cytotoxic activity, but stimulation with CA19 antigen revealed a negative association between cytotoxicity and patients' DFS (Figure 2 and Supplementary Table S1). Patients with a detectable interleukin (IL)-10 level in their sera showed a tendency towards a better OS and DFS (Supplementary Table S1). Other immune cell populations and ILs did not show prognostic capacity for patients with pancreatic carcinoma.

Immunological effects of IFN and chemotherapy. Before we started with analysis of immunological parameters for predictive purposes, we assessed effects of both therapies (immunotherapy and chemotherapy). For this purpose, the PMBS and serum from the arm A patients before the therapy with low dose of IFN (pre LDI) and 1 day after (oN LDI), and from the arm B patients before the first cycle of chemotherapy (pre 1st) and 4 weeks after for the second cycle of chemotherapy (pre 2nd) were analysed. Both therapies induced an increase in a frequency of neutrophils and monocytes in the patients' PBMC (Figure 3, both panels). IFN treatment also increased the frequency of activated and $\mathrm{CD} 21^{+}$monocytes (Figure 3, left panel). It is interesting that both treatments affected the cytotoxicity of PBMC: although the patients' IFN treatment increased the lysis of K562 cells (Figure 3, left panel), the chemotherapy reduced the cytotoxic capacity of the patients' immune cells (Figure 3, right panel). No effects of therapies were registered either on other immune cells or on cytokine levels.

Predictive immunological markers for patients with pancreatic cancer treated with chemoradioimmunotherapy. Next, we asked whether the immunological parameters measured in this study could indicate a value to predict the outcome of chemoradioimmunotherapy. For this purpose, the immunological parameters were correlated with the OS and DFS of patients from arm A (chemoradioimmunotherapy) of the CapRi-1 study.

Neutrophils are associated with a poorer OS after treatment (cut-off $49.7 \%$ of PBMC; Supplementary Table S2). Although the amount of monocytes has no effect on survival, a high CD21 expression and high amount of activated monocytes shows a tendency to be associated with better patient outcome after chemoradioimmunotherapy (cut off $56.2 \%$ of CD $21^{+}$monocytes and $48.65 \%$ of activated monocytes; Supplementary Table S2). Analysis of lymphocytes in patients' peripheral blood revealed a positive correlation between lymphocyte amount and survival after treatment (cut-off $21.6 \%$ of leukocytes; Figure 4 and Supplementary Table S2). Although a high expression of CCR7 on lymphocytes has a tendency to positively correlate with OS, a high CD21 expression on lymphocytes has a tendency to affect OS 

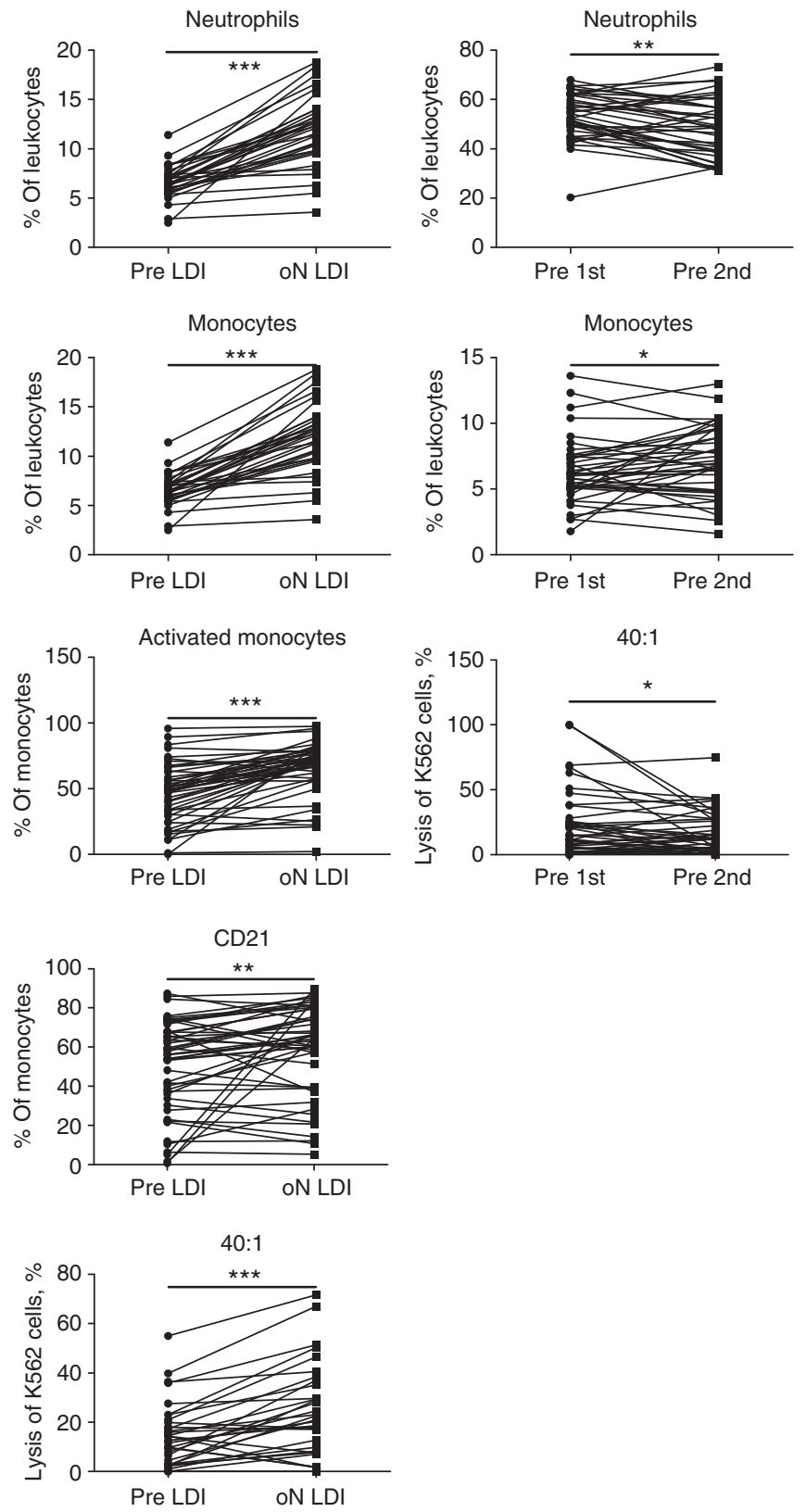

Figure 3. Immunological effects of IFN and chemotherapy. PMBS from the arm A patients before the therapy with low dose of IFN (pre LDI) and 1 day after (oN LDI), and from the arm $B$ patients before the first cycle of chemotherapy (pre 1st) and 4 weeks after for the second cycle of chemotherapy (pre 2nd) were analysed. Immunological parameters from patients treated with IFN (left panel) or with chemotherapy (right panel). ${ }^{\star} P<0.05,{ }^{*} P<0.01$ and ${ }^{* \star *} P<0.001$

negatively (cut-off $37.95 \%$ of $\mathrm{CCR}^{+}$lymphocytes and $10.2 \%$ of $\mathrm{CD} 21^{+}$lymphocytes; Supplementary Table S2).

A high frequency of naive $\mathrm{CD}^{+} \mathrm{T}$ cells shows a positive association with patient survival after chemoradioimmunotherapy (cut-off 12.65\%; Supplementary Table S2). Surprisingly, a high percentage of effector-memory (em) $\mathrm{CD}^{+} \mathrm{T}$ cells has a negative correlation with patient survival (cut-off 18.5\%; Figure 4 and Supplementary Table S2). At the same time, cytotoxic activity of $\mathrm{CD}^{+} \mathrm{T}$ cells after stimulation with MUC1 antigen showed a positive correlation with prolonged DFS compared with patients without cytotoxic activity in their PBMCs (Figure 4 and Supplementary Table S2). Although the amount of $\mathrm{CD} 4^{+} \mathrm{T}$ cells does not show to be predictive for outcome, surprisingly a high
CD152 expression on these cells correlates positive with a better DFS in treated patients (cut-off 0.5\%; Figure 4 and Supplementary Table S2). Patients with high IL-2 level in their sera showed a worse OS after chemoradioimmunotherapy (cut-off $33.6 \mathrm{pg} \mathrm{ml}^{-1}$; Figure 4 and Supplementary Table S2). Other immune cell populations and ILs did not show any predictive capacity for patients with pancreatic carcinoma treated with chemoradiotherapy in combination with IFN.

Predictive immunological markers for patients with pancreatic cancer treated with 5FU-based chemotherapy. To search for possible immunological markers for chemotherapy with 5FU, we performed a correlation analysis of immunological parameters with the OS and DFS of patients from arm B (5FU-based chemotherapy) of the CapRi-1 study.

Accumulation of $\mathrm{CD}^{+}$and $\mathrm{CD} 8{ }^{+} \mathrm{T}$ cells in tumours has been found to be predictive for DFS in this group of patients (cut-off 238 $\mathrm{CD}^{+}$cells and $162 \mathrm{CD}^{+}$cells per total area of $1 \mathrm{~mm}^{2}$; Figure 5 and Supplementary Table S3). In addition, a high amount of $\mathrm{CD}^{+}{ }^{+}$and effCD8 ${ }^{+} \mathrm{T}$ cells in the peripheral blood of patients from the chemotherapeutic arm showed a positive correlation with an improved survival (cut off $26 \%$ for $\mathrm{CD}^{+}{ }^{+} \mathrm{T}$ cells and $24.4 \%$ for $\mathrm{CD}^{+}$T cells; Figure 5 and Supplementary Table S3). Surprisingly, NK-mediated cytotoxicity from blood PBMC was negatively associated with the survival of patients treated with chemotherapy (cut-off for 1:40 target:effector $14.59 \%$ and for $1: 80,27.77 \%$ of death target cells; Figure 6 and Supplementary Table S3). Functional activity of $\mathrm{CD}^{+} \mathrm{T}$ cells showed different results depending on the antigen used for PBMC stimulation. In the case of CA19, we found that patients with an absence of granzyme B release survived longer with such a response. When MUC was used for PBMC stimulation, a positive association of granzyme B release with patients' survival was detected (Figure 6 and Supplementary Table S3). The presence of serum IL-10 correlated with improved survival of patients treated with the chemotherapy (Figure 6 and Supplementary Table S3). Other immunologic parameters did not show any predictive capacity for patients with pancreatic carcinoma treated by 5FU-based chemotherapy.

\section{DISCUSSION}

The benefit of evidence-based medicine is different in terms of the clinical course of individual patients. Therefore, oncologists aim to incorporate biomarkers in clinical routines to guide treatment algorithms (Jager et al, 2011). However, such potential biomarkers are not yet determined for cancer patients, particularly for pancreatic carcinoma. The CapRI study was a multicentre randomized phase III trial of adjuvant chemoradioimmunotherapy (Schmidt et al, 2012). The patients from this trial have undergone extensive immunomonitoring. Therefore, the main aim of this work was to validate the predictive and prognostic values of the immunological parameters investigated. Potential biomarkers for prognostic and therapy prediction properties are summarized in Figure 7.

Cytotoxic activity of $\mathrm{CD}^{+} \mathrm{T}$ cells measured by granzyme $\mathrm{B}$ release after stimulation with MUC antigen was found in this study to be predictive for the survival of patients independently of type of therapy: patients having a PBMC response to MUC stimulation indeed show a survival benefit. Based on the concept of pancreatic cancer immunity (Bazhin et al, 2013, 2014), it appears logical to assume that after surgery the micrometastases are under surveillance of the cytotoxic T cells. Besides, MUC1 is aberrantly overexpressed in $>80 \%$ of human pancreatic cancers (Hinoda et al, 2003), suggesting the presence of specific MUC1 cytotoxic T cells, which are able to police cancer progression for some time.

Now it is accepted that tumour infiltration with $\mathrm{CD} 8{ }^{+} \mathrm{T}$ cells correlates well with the survival of patients with different types of 
CD3
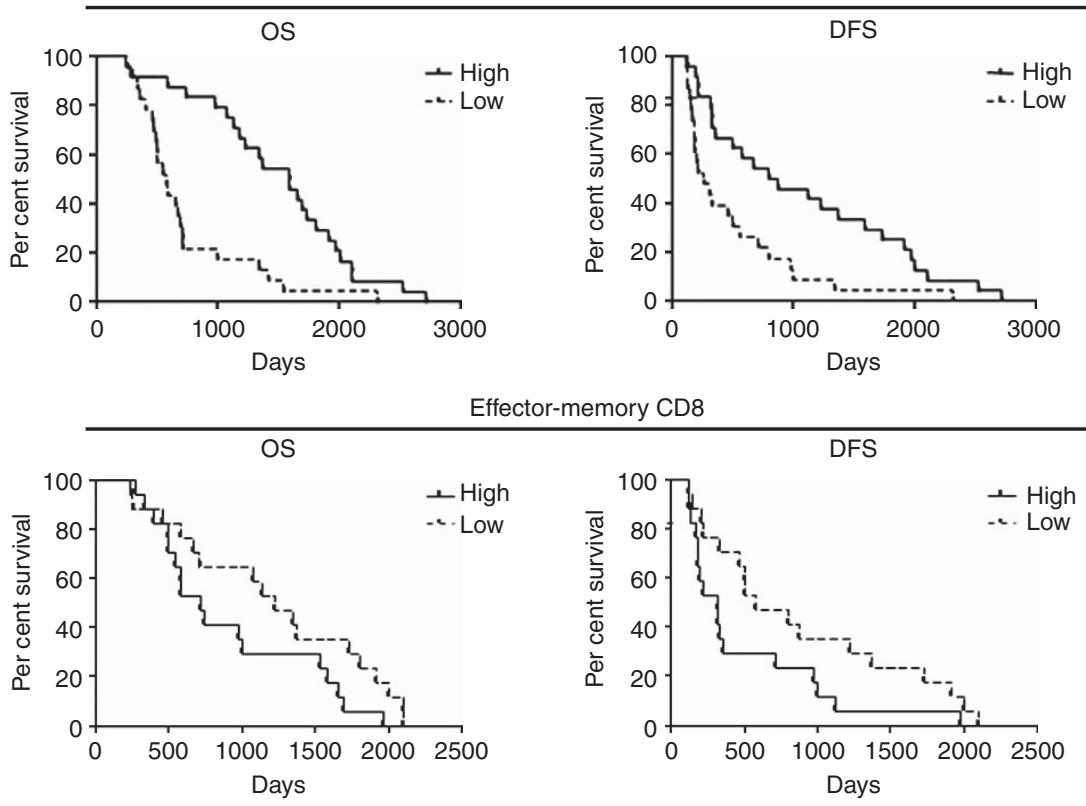

Effector-memory CD8
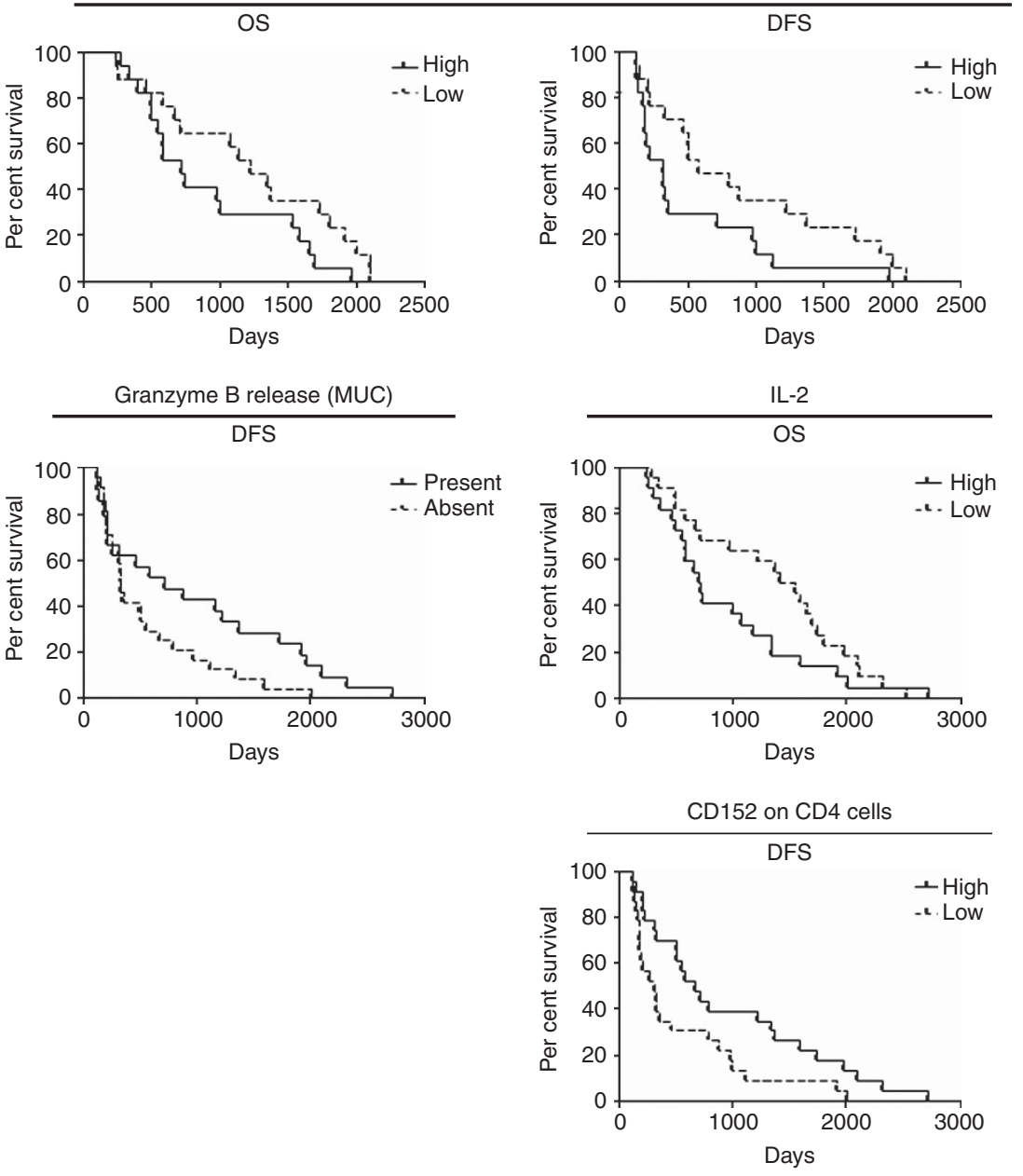

Figure 4. Immunological parameters as possible predictive markers for chemoradioimmunotherapy with IFN. Data of FACS analysis, granzyme B release and IL-2 analysis were correlated with patients' OS and/or DFS with Kaplan-Meier methods. For statistical data, see Supplementary Table S2.

cancer (Galon et al, 2006) and so can serve as an independent surrogate marker for cancer patient survival. Therefore, the tumour infiltration with immune cells may exhibit the tumour pathobiology; thus, the immune markers could in fact be a reflection of a pre-existing immune constellations and of response to a particular therapy. In our cohort of R0/R1-resected patients, we demonstrated a survival benefit for patients with high $\mathrm{CD}^{+} \mathrm{T}$ cell accumulation in their tumours. This is in line with earlier studies by Ryschich et al (2005) and Fukunaga et al (2004) who published similar observations for patients with pancreatic cancer. Importantly, we found in this study that accumulation of $\mathrm{CD} 4{ }^{+}$cells and generally $\mathrm{CD}^{+}{ }^{+}$cells correlates with a survival benefit in patients. In head and neck cancer, a survival benefit for patients with a high accumulation of $\mathrm{CD} 4^{+} \mathrm{T}$ cells in their tumours has been shown previously (Badoual et al, 2006). To our knowledge, the present study is the first report of potential predictivity of $\mathrm{CD} 3^{+} / \mathrm{CD} 4^{+}$ cell accumulation in tumours of patients with pancreatic cancer. Thus, this observation supports the hypothesis that the immune system also protects the organism against growing tumours in pancreatic cancer. In the context of therapy outcome prediction, patients from the chemotherapeutic arm showed better survival by high accumulation of $\mathrm{CD}^{+}$and $\mathrm{CD} 8^{+}$cells in their tumours. However, this was not the case for patients in the immunotherapeutic arm. This fact strengthens the immune concept of pancreatic cancer that is susceptible to immune stimulation (Bazhin et al, 2013, 2014).

The components of innate immune system -neutrophils and NK cells - were found in this study to be possible predictors of inferior survival rate in patients with pancreatic cancer. For neutrophils, however, there are data showing that this immune cell population might have a negative prognostic value (Stotz et al, 2013), whereas for NK cells such data are absent. These cells are 

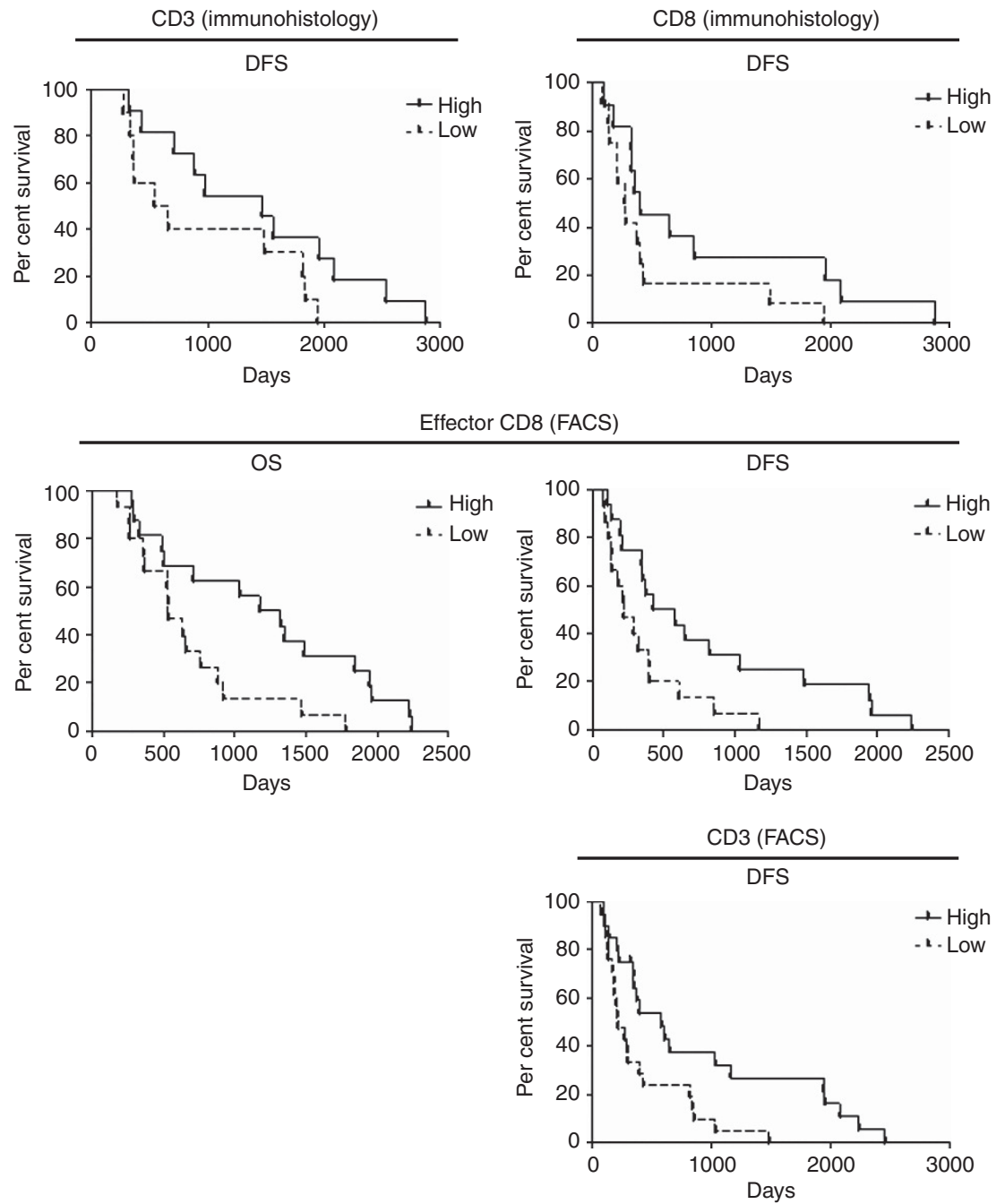

Figure 5. Immunological parameters as possible predictive markers for 5FU-based chemotherapy. Immunological parameters measured with immunohistology and FACS analysis of $\mathrm{CD} 3^{+}$lymphocytes and effCD8 ${ }^{+} \mathrm{T}^{+}$cells (Figure 5) were correlated with patients' OS and/or DFS with Kaplan-Meier methods. For statistical data, see Supplementary Table S3.

important factors in combating tumours. However, NK cells can also function as regulatory cells, interacting and controlling a variety of cell types (Pedroza-Pacheco et al, 2013). Therefore, NK cells can also limit the immune response, which might be reflected in the poor survival of patients with a high amount of NK cells in their blood. We cannot exclude that the data analysed are only an epiphenomenon, which does not however rule out a potential prognostic value of this immune cell population. Besides, although NK cells were unable to predict the survival of patients in relation to therapy, neutrophils were found to be predictive of the chemoradioimmunotherapy response but not for chemotherapy alone.

Activated monocytes produce pro-inflammatory cytokines such as TNF $\alpha$ and IL-12, and possess phagocytes and an antigenpresenting capacity (Randolph et al, 2008). Furthermore, activated monocytes express a number of co-stimulatory molecules (Van Gool et al, 1996) so that this type of immune cell has an important role in anti-cancer activity. This is supported by the observation that activated monocytes prime $\mathrm{T}$ cells in vitro and in vivo against autologous cancer (Laumbacher et al, 2012). Moreover, IFNactivated monocytes have an input in anti-tumour effects, which have also been demonstrated in earlier studies (Baron et al, 2007, 2011). Therefore, the indication of our data that activated monocytes might predict chemoradioimmunotherapy outcomes is in line with the abovementioned observations.
FACS analysis of PBMC represents the easiest means of assessing lymphocytes and their subpopulations in patients' peripheral blood, especially in relation to their potential usage as biomarkers. Although in general $\mathrm{CD}^{+}$lymphocytes were not found to be prognostic for patients' survival, these cells can predict the outcome of patients of both therapy arms. It is tempting to speculate that the high amount of $\mathrm{CD}^{+}$lymphocytes associated with a better patient prognosis is credited to 5FU-based chemotherapy, as this drug was present in both therapeutic arms. Taking into consideration the specific lymphocyte subpopulations, we found that a high amount of effCD ${ }^{+} \mathrm{T}$ cells in the peripheral blood of the pancreatic carcinoma patients could have a positive prognostic value. It is not surprising that a patient with a high amount of effCD8 ${ }^{+} \mathrm{T}$ cells has a better survival rate, as these cells represent the foreground of anti-cancer defence (Hadrup et al, 2013). However, this is a first observation of a potential prognostic value of effCD8 ${ }^{+} \mathrm{T}$ cells in the peripheral blood of pancreatic cancer patients. Importantly, the percentage of effCD8 ${ }^{+} \mathrm{T}$ cells can also predict the outcome of chemotherapy but not of chemoradioimmunotherapy, making this cell population a potential predictive marker. Interestingly, a high percentage of naive $\mathrm{CD}^{+} \mathrm{T}$ cells predicts an increased survival rate of patients treated with the combined therapy. It is well known that IFN is one of the necessary signals providing development of the eff/em phenotypes by $\mathrm{CD} 8{ }^{+} \mathrm{T}$ cells. Therefore, we speculate that naive $\mathrm{CD} 8^{+} \mathrm{T}$ cells 

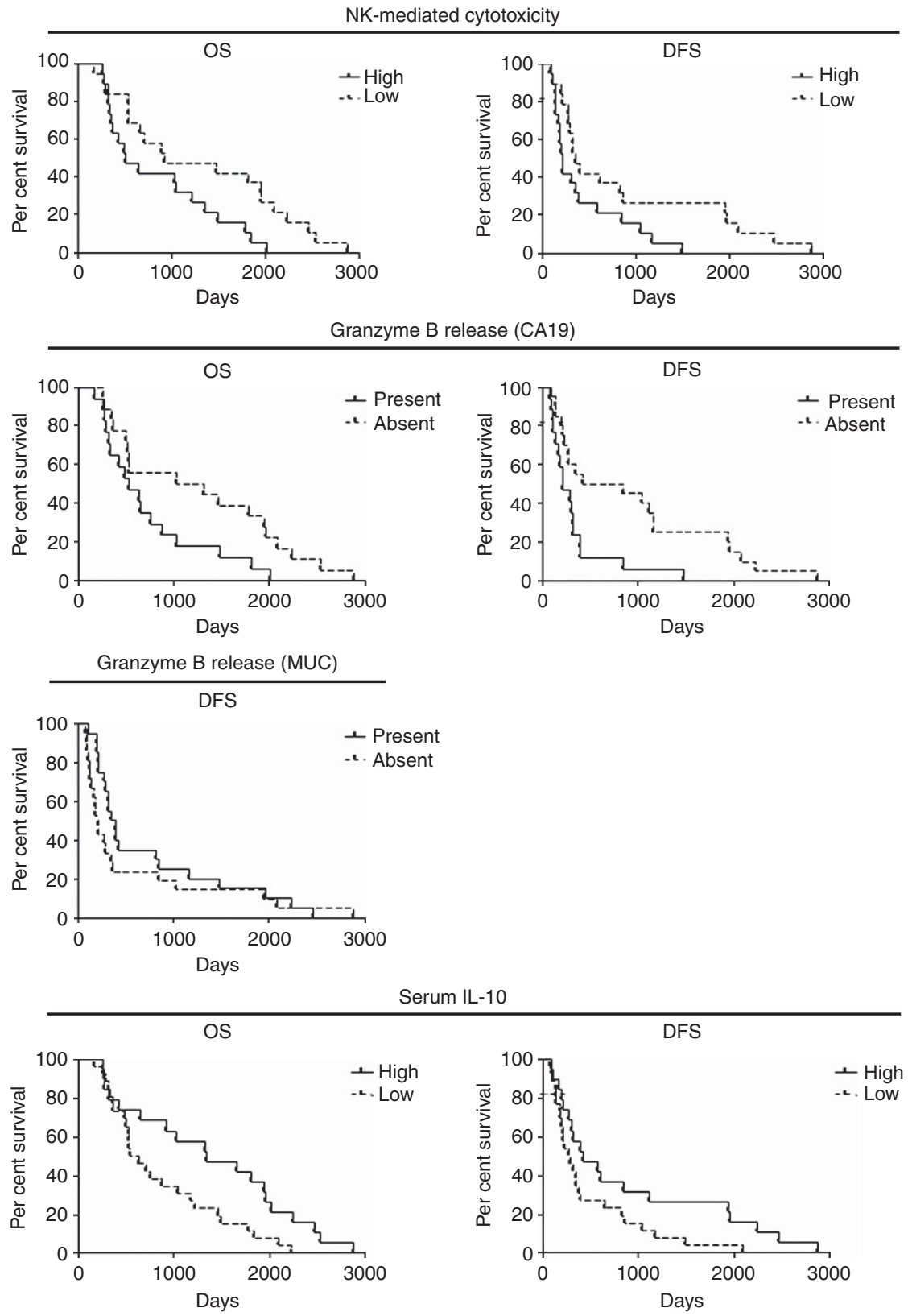

Figure 6. Immunological parameters as possible predictive markers for 5FU-based chemotherapy. NK-mediated cytotoxicity or data of granzyme $B$ release and of IL-10 analysis (Figure 5) were correlated with patients' OS and/or DFS with Kaplan-Meier methods. For statistical data, see Supplementary Table S3.

before chemoradioimmunotherapy can acquire eff/em phenotypes, leading to improved immune surveillance of micrometastases still existing after surgery, finally manifesting in a better therapy outcome. As in arm B we did not find such a correlation, this effect can indeed be linked to IFN. Another surprising fact is that in our study the high amount of emCD8 ${ }^{+} \mathrm{T}$ cells predicted a poor outcome of the combined therapy. However, these emCD ${ }^{+}$ $\mathrm{T}$ cells are determined to be on the periphery (in PBMC) and not in the tumour. Therefore, it could be that high amounts of these cells in the blood correlates with a low amount of emCD8 ${ }^{+} \mathrm{T}$ cells in tumours. The same explanation could be applied to our observation that high CD152 expression of CD4 ${ }^{+}$T cells, which may have a regulatory $\mathrm{T}$-cell phenotype, correlates with a better survival of patients from the chemoradioimmunotherapy arm. Here we also argue that a high amount of such cells on the periphery reflects a low amount of immunosuppressive cells on the tumour side. Otherwise, the expression of this molecule could control undesirable overwhelming inflammation, which in turn might be beneficial for patients. Nevertheless, these hypotheses need to be verified in future trials.

In our study, high frequency of $\mathrm{CCR} 7^{+}$lymphocytes has a tendency to positively correlate with OS in chemoradioimmunotherapy arm. Indeed, CCR7 expression is an important characteristic of naive and especially central memory $\mathrm{T}$ lymphocytes. Importantly, CCR7, in addition to other properties, can control the migration of memory $\mathrm{T}$ cells to and within secondary lymphoid organs, such as lymph nodes, and thus make additional input into APC maturation (Forster et al, 2008). We can assume that during chemoradioimmunotherapy, IFN injection results in an unspecific stimulation leading to an increase among monocytes and APC. Central memory CD8 $\mathrm{T}$ cells (CCR7 positive), which are the result of the first confrontation of the immune system with the tumour, are re-stimulated and increased in their numbers, resulting in even more pronounced numbers of CCR7-positive cells. Central memory cells 


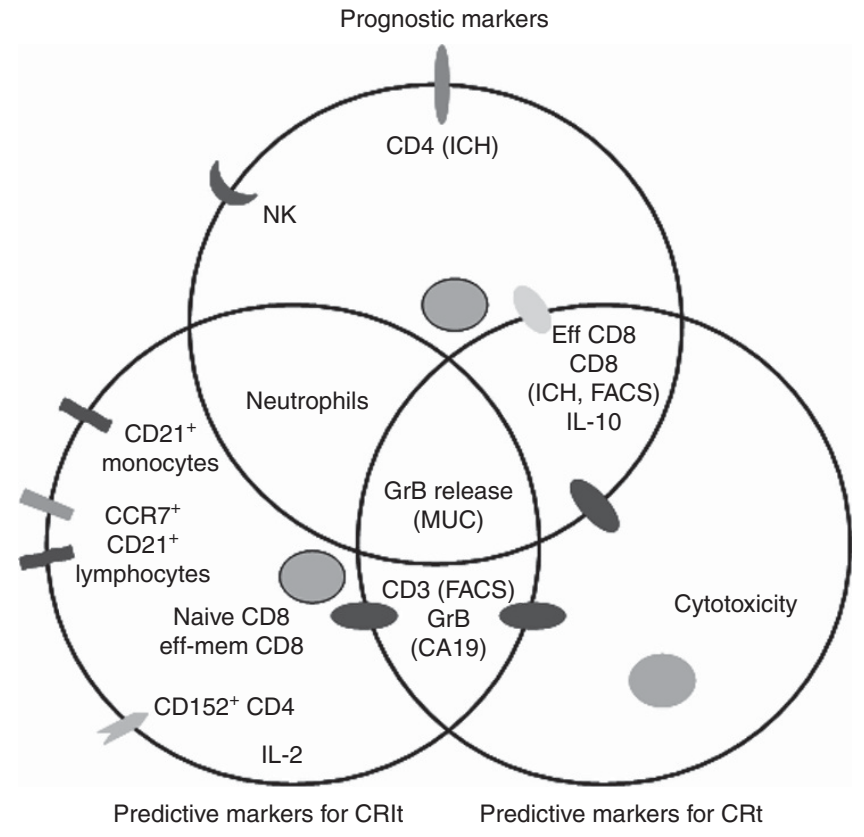

Figure 7. Summary of immunological parameters found to be potential prognostic markers for patients with pancreatic cancer and potential predictive markers for chemoradioimmunotherapy or 5FU-based chemotherapy. CRIt = chemoradioimmunotherapy; $\mathrm{Ct}=$ chemotherapy.

are known to exhibit direct cytotoxicity and proliferation potential after re-stimulation (Sallusto et al, 2004). Moreover, together with increased pool of em cells, they are able to produce eff cytokines. This all will result in increased immune response. Chemoradiotherpy in turn additionally provide the release of tumour antigens together with danger signal, leading to the specific immune stimulation. Taking together the CCR7 expression in our case probably reflects the amount of central memory $\mathrm{T}$ cells and their ability to migrate, whereby both could lead to the promotion of anti-tumour immunity and thus increased survival of patients.

In respect to $\mathrm{CD} 21$, this complement receptor type 2 can interact additionally with the low-affinity receptor for IgE (CD23) and bind IFN. It has an important role in the activation and proliferation of B cells. Usually, B cells expressed considerable amounts of CD21 on their cell surface, despite the expression of CD21 described for T cells as well (Zabel and Weis, 2001). Role of CD21 on T cell is not fully understood but it was shown that CD21 might increase adherence of $\mathrm{T}$ cells to nucleated target cells bearing C3d and this function may be relevant to cytotoxicity or other T-cell activities requiring cell-cell interaction (Levy et al, 1992). In our case, a high CD21 expression on lymphocytes has a tendency to affect OS negatively. Hence, we could assume the following reasons for this: (1) increased expression of CD21 results in modulated activation of B cells or different adherence capacity of T cells; (2) moreover and most probably, this CD21 expression makes the lymphocytes in general more sensitive to the IFN. In this case, direct effect of IFN on lymphocytes could result in their overstimulation, which can be contra productive for the survival of patients, especially in context of chemotherapy. In line, we in the CapRI2 (Karakhanova et al, 2014) study and the others observed a decrease in total leukocytes number but increase in monocytes after IFN therapy. In addition, IFN might initiate the expression of several genes in CD21 + lymphocytes, which also could affect the survival. Nevertheless, which factor and how exactly it can affect survival of the patients through increased CD21 expression on lymphocytes need to be investigated further.
Serum IL-10 amount was found to be prognostic for patients with pancreatic cancer independent of the therapy carried out, and at the same time is predictive for outcome of chemotherapy but not of combined therapy with IFN. IL-10 is an immunosuppressive factor that has been found to be produced at the tumour site and to be increased in the serum of cancer patients (Galizia et al, 2002; Chan et al, 2012). However, in our study, patients with detectable IL-10 had a better survival rate compared with patients without IL-10 in their sera. We do not know what the basis of the abovementioned difference is, but it cannot be excluded that this effect is cancer-type specific, especially for pancreatic cancer, which is strongly immunosuppressive (Bazhin et al, 2014).

Immunological parameters identified in this trial as possible surrogate markers may be of interest and importance in the context of personalized medicine for the improvement of pancreatic cancer treatment after validation in a prospective trial.

\section{ACKNOWLEDGEMENTS}

We thank Mr Markus Herbst for his excellent technical assistance. We gratefully acknowledge Professor Angela Märten for the study initiation. This work was supported partly by a grant from Manfred Lautenschläger Foundation.

\section{CONFLICT OF INTEREST}

The authors declare no conflict of interest.

\section{REFERENCES}

Badoual C, Hans S, Rodriguez J, Peyrard S, Klein C, Agueznay Nel H, Mosseri V, Laccourreye O, Bruneval P, Fridman WH, Brasnu DF, Tartour E (2006) Prognostic value of tumor-infiltrating CD4 + T-cell subpopulations in head and neck cancers. Clin Cancer Res 12(2): 465-72.

Baron S, Finbloom J, Horowitz J, Bekisz J, Morrow A, Zhao T, Fey S, Schmeisser H, Balinsky C, Miyake K, Clark C, Zoon K (2011) Near eradication of clinically relevant concentrations of human tumor cells by interferon-activated monocytes in vitro. J Interferon Cytokine Res 31(7): $569-73$.

Baron S, Hernandez J, Bekisz J, Poast J, Goldman N, Clouse K, Fields K, Bacot S, Wang J, Zoon K (2007) Clinical model: interferons activate human monocytes to an eradicative tumor cell level in vitro. J Interferon Cytokine Res 27(2): 157-63.

Bazhin AV, Bayry J, Umansky V, Werner J, Karakhanova S (2013) Overcoming immunosuppression as a new immunotherapeutic approach against pancreatic cancer. Oncoimmunology 2(9): e25736.

Bazhin AV, Shevchenko I, Umansky V, Werner J, Karakhanova S (2014) Two immune faces of pancreatic adenocarcinoma: possible implication for immunotherapy. Cancer Immunol Immunother 63(1): 59-65.

Bellati F, Visconti V, Napoletano C, Antonilli M, Frati L, Panici PB, Nuti M (2009) Immunology of gynecologic neoplasms: analysis of the prognostic significance of the immune status. Curr Cancer Drug Targets 9(4): 541-65.

Chan SL, Mo FK, Wong CS, Chan CM, Leung LK, Hui EP, Ma BB, Chan AT, Mok TS, Yeo W (2012) A study of circulating interleukin 10 in prognostication of unresectable hepatocellular carcinoma. Cancer 118(16): 3984-92.

Conroy T, Desseigne F, Ychou M, Bouche O, Guimbaud R, Becouarn Y, Adenis A, Raoul JL, Gourgou-Bourgade S, de la Fouchardiere C, Bennouna J, Bachet JB, Khemissa-Akouz F, Pere-Verge D, Delbaldo C, Assenat E, Chauffert B, Michel P, Montoto-Grillot C, Ducreux M (2011) FOLFIRINOX versus gemcitabine for metastatic pancreatic cancer. N Engl J Med 364(19): 1817-25.

Copier J, Whelan M, Dalgleish A (2006) Biomarkers for the development of cancer vaccines: current status. Mol Diagn Ther 10(6): 337-43. 
Cress RD, Yin D, Clarke L, Bold R, Holly EA (2006) Survival among patients with adenocarcinoma of the pancreas: a population-based study (United States). Cancer Causes Control 17(4): 403-9.

Forster R, Davalos-Misslitz AC, Rot A (2008) CCR7 and its ligands: balancing immunity and tolerance. Nat Rev Immunol 8(5): 362-71.

Fukunaga A, Miyamoto M, Cho Y, Murakami S, Kawarada Y, Oshikiri T, Kato K, Kurokawa T, Suzuoki M, Nakakubo Y, Hiraoka K, Itoh T, Morikawa T, Okushiba S, Kondo S, Katoh H (2004) CD8 + tumor-infiltrating lymphocytes together with $\mathrm{CD} 4+$ tumor-infiltrating lymphocytes and dendritic cells improve the prognosis of patients with pancreatic adenocarcinoma. Pancreas 28(1): e26-31.

Galizia G, Orditura M, Romano C, Lieto E, Castellano P, Pelosio L, Imperatore V, Catalano G, Pignatelli C, De Vita F (2002) Prognostic significance of circulating IL-10 and IL-6 serum levels in colon cancer patients undergoing surgery. Clin Immunol 102(2): 169-78.

Galon J, Costes A, Sanchez-Cabo F, Kirilovsky A, Mlecnik B, Lagorce-Pages C, Tosolini M, Camus M, Berger A, Wind P, Zinzindohoue F, Bruneval P, Cugnenc PH, Trajanoski Z, Fridman WH, Pages F (2006) Type, density, and location of immune cells within human colorectal tumors predict clinical outcome. Science 313(5795): 1960-4.

Gogas H, Kirkwood JM (2009) Predictors of response to interferon therapy. Curr Opin Oncol 21(2): 138-43.

Hadrup S, Donia M, Thor Straten P (2013) Effector CD4 and CD8 T cells and their role in the tumor microenvironment. Cancer Microenviron 6(2): 123-33.

Hinoda Y, Ikematsu Y, Horinochi M, Sato S, Yamamoto K, Nakano T, Fukui M, Suehiro Y, Hamanaka Y, Nishikawa Y, Kida H, Waki S, Oka M, Imai K, Yonezawa S (2003) Increased expression of MUC1 in advanced pancreatic cancer. J Gastroenterol 38(12): 1162-6.

Jager D, Seil I, Halama N (2011) Predictive immunological markers in oncology. Front Immunol 2: 86.

Jimeno A, Hidalgo M (2006) Molecular biomarkers: their increasing role in the diagnosis, characterization, and therapy guidance in pancreatic cancer. Mol Cancer Ther 5(4): 787-96.

Karakhanova S, Mosl B, Harig S, Ahn K, Fritz J, Schmidt J, Jager D, Werner J, Bazhin AV (2014) Influence of interferon-alpha combined with chemo (radio) therapy on immunological parameters in pancreatic adenocarcinoma. Int J Mol Sci 15(3): 4104-25.

Knaebel HP, Marten A, Schmidt J, Hoffmann K, Seiler C, Lindel K, Schmitz-Winnenthal H, Fritz S, Herrmann T, Goldschmidt H, Krempien R, Mansmann U, Debus J, Diehl V, Buchler MW (2005) Phase III trial of postoperative cisplatin, interferon alpha-2b, and 5-FU combined with external radiation treatment versus 5-FU alone for patients with resected pancreatic adenocarcinoma - CapRI: study protocol [ISRCTN62866759]. BMC Cancer 5: 37.

Lake RA, Robinson BW (2005) Immunotherapy and chemotherapy-a practical partnership. Nat Rev Cancer 5(5): 397-405.

Laumbacher B, Gu S, Wank R (2012) Activated monocytes prime naive T cells against autologous cancer: vigorous cancer destruction in vitro and in vivo. Scand J Immunol 75(3): 314-28.

Levy E, Ambrus J, Kahl L, Molina H, Tung K, Holers VM (1992) T lymphocyte expression of complement receptor 2 (CR2/CD21): a role in adhesive cell-cell interactions and dysregulation in a patient with systemic lupus erythematosus (SLE). Clin Exp Immunol 90(2): 235-44.

Neoptolemos JP, Stocken DD, Bassi C, Ghaneh P, Cunningham D, Goldstein D, Padbury R, Moore MJ, Gallinger S, Mariette C, Wente MN, Izbicki JR, Friess H, Lerch MM, Dervenis C, Olah A, Butturini G, Doi R, Lind PA, Smith D, Valle JW, Palmer DH, Buckels JA, Thompson J, McKay CJ, Rawcliffe CL, Buchler MW (2010) Adjuvant chemotherapy with fluorouracil plus folinic acid vs gemcitabine following pancreatic cancer resection: a randomized controlled trial. JAMA 304(10): 1073-81.

Oettle H, Post S, Neuhaus P, Gellert K, Langrehr J, Ridwelski K, Schramm H, Fahlke J, Zuelke C, Burkart C, Gutberlet K, Kettner E, Schmalenberg H, Weigang-Koehler K, Bechstein WO, Niedergethmann M, Schmidt-Wolf I, Roll L, Doerken B, Riess H (2007) Adjuvant chemotherapy with gemcitabine vs observation in patients undergoing curative-intent resection of pancreatic cancer: a randomized controlled trial. JAMA 297(3): 267-77.

Pedroza-Pacheco I, Madrigal A, Saudemont A (2013) Interaction between natural killer cells and regulatory $\mathrm{T}$ cells: perspectives for immunotherapy. Cell Mol Immunol 10(3): 222-9.

Randolph GJ, Jakubzick C, Qu C (2008) Antigen presentation by monocytes and monocyte-derived cells. Curr Opin Immunol 20(1): 52-60.

Ryschich E, Notzel T, Hinz U, Autschbach F, Ferguson J, Simon I, Weitz J, Frohlich B, Klar E, Buchler MW, Schmidt J (2005) Control of T-cellmediated immune response by HLA class I in human pancreatic carcinoma. Clin Cancer Res 11(2 Pt 1): 498-504.

Sallusto F, Geginat J, Lanzavecchia A (2004) Central memory and effector memory T cell subsets: function, generation, and maintenance. Annu Rev Immunol 22: 745-63.

Schmidt J, Abel U, Debus J, Harig S, Hoffmann K, Herrmann T, Bartsch D, Klein J, Mansmann U, Jager D, Capussotti L, Kunz R, Buchler MW (2012) Open-label, multicenter, randomized phase III trial of adjuvant chemoradiation plus interferon Alfa- $2 \mathrm{~b}$ versus fluorouracil and folinic acid for patients with resected pancreatic adenocarcinoma. J Clin Oncol 30(33): 4077-83.

Schmidt J, Jager D, Hoffmann K, Buchler MW, Marten A (2007) Impact of interferon-alpha in combined chemoradioimmunotherapy for pancreatic adenocarcinoma (CapRI): first data from the immunomonitoring. $J$ Immunother 30(1): 108-15.

Simon R (2008) The use of genomics in clinical trial design. Clin Cancer Res 14(19): 5984-93.

Simon R, Altman DG (1994) Statistical aspects of prognostic factor studies in oncology. Br J Cancer 69(6): 979-85.

Stotz M, Gerger A, Eisner F, Szkandera J, Loibner H, Ress AL, Kornprat P, AlZoughbi W, Seggewies FS, Lackner C, Stojakovic T, Samonigg H, Hoefler G, Pichler M (2013) Increased neutrophil-lymphocyte ratio is a poor prognostic factor in patients with primary operable and inoperable pancreatic cancer. Br J Cancer 109(2): 416-21.

Van Gool SW, Vandenberghe P, de Boer M, Ceuppens JL (1996) CD80, CD86 and CD40 provide accessory signals in a multiple-step T-cell activation model. Immunol Rev 153: 47-83.

Von Hoff DD, Ervin T, Arena FP, Chiorean EG, Infante J, Moore M, Seay T, Tjulandin SA, Ma WW, Saleh MN, Harris M, Reni M, Dowden S, Laheru D, Bahary N, Ramanathan RK, Tabernero J, Hidalgo M, Goldstein D, Van Cutsem E, Wei X, Iglesias J, Renschler MF (2013) Increased survival in pancreatic cancer with nab-paclitaxel plus gemcitabine. $N$ Engl J Med 369(18): 1691-703.

Zabel MD, Weis JH (2001) Cell-specific regulation of the CD21 gene. Int Immunopharmacol 1(3): 483-93.

This work is published under the standard license to publish agreement. After 12 months the work will become freely available and the license terms will switch to a Creative Commons AttributionNonCommercial-Share Alike 4.0 Unported License.

Supplementary Information accompanies this paper on British Journal of Cancer website (http://www.nature.com/bjc) 